\section{Seoju Hwangkeum Sol and Hwangsim Sol Black Pine Cultivars}

\author{
Jeong-Ho Lee ${ }^{1}$ \\ Useful Plant Resources Center, Korea National Arboretum, 21-10, Dudamgil, \\ Yongmum-myeon, Yangpyeong, Gyeonggi-do 476-845, South Korea
}

Sugwang Lee

National Institute of Forest Science, Division of Special-purpose Trees, 39-Onjeng-ro, Gwonseon-gu, Suwon, South Korea

\section{Yong-Hee Yu \\ Seoju Plantation, 159 Sungju-ri, Janghang-eup, Seoncheon-gun, Chungnam, South Korea}

Additional index words. ornamental plant, topiary, bonsai, plant introduction, conifer, Pinus

The evergreen Korean black pine (Pinus thunbergii L., Pinaceae) is distributed in hardiness zones 5-10 (Magarey et al., 2008). Black pine is a garden and bonsai tree in Korea and Japan, as it can be trained and has many latent buds (Lee, 1999). Annual $P$. thunbergii bonsai sales has been $\approx 4$ million dollars in South Korea (Korea Forest Service, 2013). There are currently 16 cultivars: Compacta, Globosa, Lseli, Majestic Beauty, Mini Mounds, Mt. Hood Prostrate, Oculusdraconis, Pygmaea, Thunderhead, Banshosho, Tailored, Green Elf, Shirome Janome, Ansu Hwanggumsong, Ansu Hobansong, and Ansu Migumsong, which originated from Japanese black pine (Richard, 2007). The new cultivar, Seoju Hwangkeum Sol with yellow leaves and shoots was selected from Seoju plantation at Seocheon-gun Seongjuri, Chungnam Province, South Korea, in 2001. Another new cultivar Hwangsim Sol was selected from a 25 -year-old population of $P$. thunbergii at Haman-gun Mukiri, Gyeongnam Province, South Korea, in 2002. This report describes these two new cultivars.

\section{Origin}

In 2002, 'Seoju Hwangkeum Sol' (AP 010201; PI 250450) sapling with yellow leaves and shoots was selected from a 25-year-old population of $P$. thunbergii [4 m height and $15 \mathrm{~cm}$ diameter at breast height $(\mathrm{DBH})]$ that was planted in the Seocheongun Seongjuri, Chungnam Province, South Korea, in 1976.

We selected 80 scions from the original sapling during the period 2002-09 and grafted the selection to a 4-year-old $P$. thunbergii rootstock. These grafts were repeated annually, resulting in 100 grafted plants by 2009 , and we planted them in fields at the 'Seoju' Plantation nursery. Selections

Received for publication 16 June 2015. Accepted for publication 16 Nov. 2015.

${ }^{1}$ Corresponding author. E-mail: mtmac@forest.go.kr.
In 2000, 'Hwangkeum Sol' (AP 010201; PI 250450) sapling with yellow leaves and shoots was selected from a 25 -year-old population of $P$. thunbergii ( $6 \mathrm{~m}$ height and $8.7 \mathrm{~cm} \mathrm{DBH}$ ) that was planted in the Hamangun Mukiri, Gyeongnam Province, South Korea, in 1976. We selected 500 scions from the original sapling during the period 200407 and grafted the selection to a 4-year-old $P$. thunbergii rootstock. These grafts were repeated annually resulting in 500 grafted plants that accumulated by 2010 . They were subsequently planted in fields in the 'Seoju' Plantation nursery.

In 2004, selections were made by Y.H. You in replicated field trials. Currently, we have 500 'Hwangsim Sol' plants from both 17-year-old grafts and 11- to 13-year-old individuals. They have the same uniform characteristics as the parent tree. We have also filed PVP application for protection (No. 23).

\section{Description}

initially made from replicated field trials conducted by Y.H. You in 2002. Currently, we have 100 'Seoju Hwangkeum Sol' plants, from 11-year-old grafts and 5- to 8 -year-old individuals. They have the same uniform characteristics as the parent tree. A plant variety protection (PVP) application has been filed (No. 22).
Main characteristics. 'Seoju Hwangkeum
Sol' (AP 010201; PI 250450) is a 25 -year-old
arrow-leaved evergreen tree $4 \mathrm{~m}$ high, with
DBH of $20 \mathrm{~cm}$ and a crown width of $5 \mathrm{~m}$
Fig. 1). Mature leaves are $12.5 \mathrm{~cm}$ long
and $1.6 \mathrm{~mm}$ wide, which are smaller than
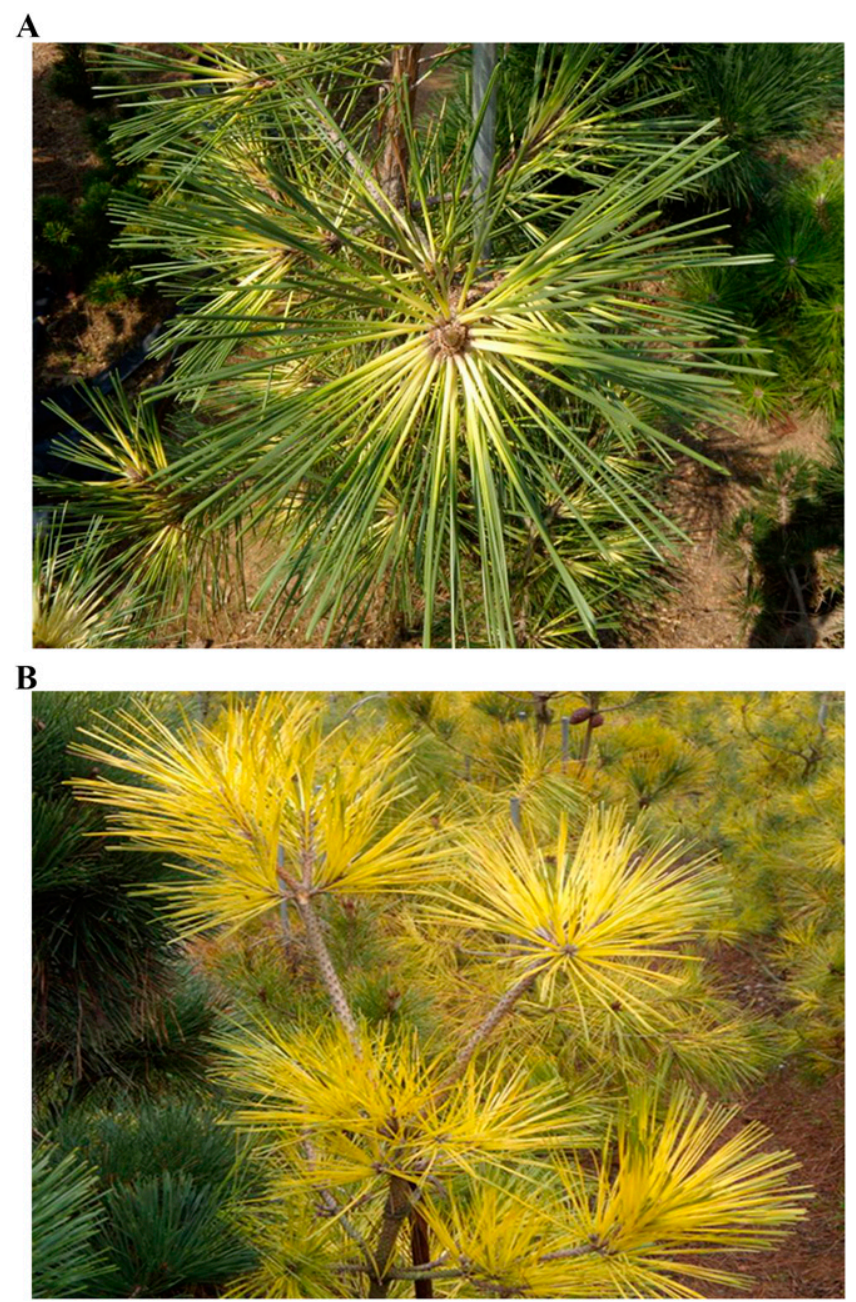

Fig. 1. (A) Pinus 'Seoju Hwangkeum Sol' (B) and 'Hwangsim Sol'. 
P. thunbergii leaves that are typically $17.3 \mathrm{~cm}$ long and $1.4 \mathrm{~mm}$ wide. Half of the 'Seoju Hwangkeum Sol' leaf was white-green and the rest was lemon. Yellow patterns were expressed from the basal part of the leaf to half the length of the leaf. The leaf color was pale yellow in early spring and increasingly yellow with growth. From spring to summer, the green part became increasingly deeper green compared with $P$. thunbergii. New leaves appeared in May and June, and their color was in the "yellow orange" International Union for the Protection of New Varieties of Plants (UPOV) color group (UCG, UPOV, 2013) corresponding to the Royal Horticultural Society (RHS) Color Chart (Royal Horticultural Society, 2007) reference numbers "013A" and "013B." Shoots from winter buds were in the "medium green" to "light green" UCG (RHS 142A-B). Shoots were greener (RHS 150 A-B) in the summer, but the color was brighter green compared with the cultivar Ansu Hwanggumsong. Leaf edge turned yellow by the end of August and whole leaves rapidly turned yellow after frost. From October to April, leaf color remained in the "yellow orange" UCG (RHS 017 A-B) compared with $P$. thunbergii. A strong characteristic of 'Seoju Hwangkeum Sol' was clear yellow leaves ["yellow orange" UCG (RHS 013 A-B)] by mid-May.

'Hwangsim Sol' (AP 020501; PI 250645) is a 14-year-old narrow-leaved evergreen tree that is $6 \mathrm{~m}$ height with a DBH of $87 \mathrm{~cm}$ and a crown width of $6 \mathrm{~m}$ (Fig. 1). Mature leaves are $11.3 \mathrm{~cm}$ long and $1.5 \mathrm{~mm}$ wide, which are smaller than normal leaves of $P$. thunbergii (leaves $17.3 \mathrm{~cm}$ long and $1.4 \mathrm{~mm}$ wide). Yellow patterns and seasonal characteristics of 'Hwangsim Sol' leaves color were golden yellow during 9 months and pale yellow and green during the remaining 3 month of a year. Basal leaves of 2- or 3-year-old plants were red, which conferred very high ornamental value. New leaves appeared in May and June. They were in the "yellow" UCG (RHS 011 A) and became increasingly yellow over time. Young shoots from May to June fell into the "light yellow" and "light yellow orange" UCG (RHS $011 \mathrm{C}-\mathrm{D}$ ), and then changed to green from the inside to the outside of the shoots in July.

Culture. 'Seoju Hwangkeum Sol' and 'Hwangsim Sol' grew well in the shade and in well-drained soil with loamy sand. In April, a 9-cm side veneer graft was tied to rootstock for vegetative propagation (Toogood, 1999).

'Seoju Hwangkeum Sol' was better adapted to $50 \%$ shade than to bright sun. If trees were to grow strongly in bright sunlight, needles could burn from the outside to inside and young trees should be grown in 50\% shade. This cultivar did not recover well after pruning. One of the most distinguishing characteristics of 'Seoju Hwangkeum Sol' was the continuous yellow expression of leaves compared with yellowing from March to October of the control variety 'Ansu Hwanggumsong'.

'Hwangsim Sol' was adapted to $30 \%$ shade than bright sunlight. One of the best characteristics of 'Hwangsim Sol' was the yellow expression of leaves during 9 months of the year. Secondary shoot grew well after primary shoots were cut in May. Leaves displayed a stronger yellow color in response to organic fertilizers compared with their response to chemical fertilizer application.

\section{Availability}

Like other woody ornamentals in 'Seoju' Plantation, 'Seoju Hwangkeum Sol' and 'Hwangsim Sol' are entitled to protection as varieties and cannot be propagated and sold freely, but these plants are available for wholesale, by mail order, and from a few retail nurseries. 'Seoju' Plantation has stocks of these cultivars available for general distribution.

\section{Literature Cited}

Korea Forest Service. 2013. Production of Forest Products (2012). Korea Forest Service, Daejeon, Korea.

Lee, T.B. 1999. Illustrated Flora of Korea. Hyangmunsa, Seoul, Korea.

Magarey, R.D., D.M. Borchert, and J.W. Schlegel. 2008. Global plant hardiness zones for phytosanitary risk analysis. Scientia Agricola. 65:54-59.

Richard, L.B. 2007. Conifers for gardens: An illustrated encyclopedia, p. 317-319. Timber Press, Portland, OR.

Royal Horticultural Society (RHS). 2007. RHS colour chart. Royal Horticultural Society, London, UK.

Toogood, A.R. 1999. Plant propagation. American Horticultural Society, New York, NY.

UPOV. 2013. Glossary of terms used in UPOV documents. International Union for the Protection of New Varieties of Plants, Geneva, Switzerland. 25 Sept. 2015. <http://www.upov.int/ edocs/tgpdocs/en/tgp_14.pdfs. 